Article

\title{
Non-Parametric Threshold Estimation for the Wiener-Poisson Risk Model
}

\author{
Honglong You ${ }^{1, *(\mathbb{D})}$ and Yuan Gao ${ }^{2}$ \\ 1 School of Statistics, Qufu Normal University, Qufu 273165, China \\ 2 School of Mathematics, Qufu Normal University, Qufu 273165, China; gyyq1413@163.com \\ * Correspondence: yougaoyou815@qfnu.edu.cn
}

Received: 15 April 2019; Accepted: 27 May 2019; Published: 3 June 2019

\begin{abstract}
In this paper, we consider the Wiener-Poisson risk model, which consists of a Wiener process and a compound Poisson process. Given the discrete record of observations, we use a threshold method and a regularized Laplace inversion technique to estimate the survival probability. In addition, we also construct an estimator for the distribution function of jump size and study its consistency and asymptotic normality. Finally, we give some simulations to verify our results.
\end{abstract}

Keywords: Wiener-Poisson risk model; survival probability; Nonparametric threshold estimation

\section{Introduction}

Let $S=\left\{S_{t}\right\}_{t \geq 0}$ with $S_{0}=0$ be a compound Poisson process defined as

$$
S_{t}=\sum_{i=1}^{N_{t}} \gamma_{i}, \quad t \geq 0
$$

where $\left\{N_{t}\right\}_{t \geq 0}$ is a Poisson process with unknown intensity $\lambda>0$, and $\gamma_{1}, \gamma_{2}, \gamma_{3}, \ldots$ are independent and identically distributed positive sequence of random variables with unknown distribution function $F$ supported on $(0, \infty)$.

The Wiener-Poisson risk process is defined by

$$
X_{t}=x+c t+\sigma W_{t}-\sum_{i=1}^{N_{t}} \gamma_{i}, \quad t \geq 0,
$$

where $x$ is a given positive constant, $\sigma>0$ is an unknown constant, the corresponding process $\left\{N_{t}, t \geq 0\right\}$ is called the claim number process, $\left\{\gamma_{i}\right\}_{i=1,2, \ldots}$ is a sequence of claims, and $\left\{W_{t}\right\}_{t \geq 0}$ is a standard Brownian motion. Suppose that $\left\{N_{t}\right\}_{t \geq 0},\left\{W_{t}\right\}_{t \geq 0}$ and $\left\{\gamma_{i}\right\}_{i=1,2, \ldots}$ are independent of each other, and the mean and variance of claim are finite, i.e., $\mu_{\gamma}=\int_{0}^{\infty} x F(d x)<\infty, \sigma_{\gamma}^{2}=$ $\int_{0}^{\infty} x^{2} F(d x)-\mu_{\gamma}^{2}<\infty$. Further, we assume that the risk model in Equation (1) has a relative safety loading $\omega=\frac{c}{\lambda \mu_{\gamma}}-1>0$. Let $\tau(x)=\inf \left\{t>0 ; X_{t} \leq 0, X_{0}=x\right\}$. The survival probability of the risk model in Equation (1) is defined by:

$$
\Phi(x)=\mathbf{P}(\tau(x)=\infty),
$$

and $\Psi(x)=1-\Phi(x)$, the probability of ruin.

In the last few decades, many works have been contributed to the survival probability for the risk model in Equation (1) and its extended risk model. In [1], the author first introduced the risk model in Equation (1) and established an asymptotic estimate for $\Psi(x)$. In [2], the authors showed that $\Phi(x)$ satisfies a defective renewal equation. By renewal theory, they obtained the Pollaczeck-Khinchin 
formula of $\Phi(x)$. Accurate calculation and approximation for $\Psi(x)$ has always been an inspiration and an important source of technological development for actuarial mathematics (see, e.g., [3-9]). Although various approximations to the probability of ruin (e.g., importance sampling or saddle-point approximations) are now available, developing alternative approximations of different nature is still an interesting and practical problem.

In recent years, many authors studied the ruin probability by using statistical methods (see, e.g., [10-18]). In [17], the author assumed that $\left\{X_{t_{i}^{n}} \mid t_{i}^{n}=i h_{n} ; i=0,1,2, \ldots, n\right\}$ and $\left\{\gamma_{1}, \gamma_{2}, \ldots, \gamma_{N_{t_{n}^{n}}}\right\}$ are observed, where $h_{n}=t_{i}^{n}-t_{i-1}^{n}$ is the sampling interval and the time of claims are known. The author constructed an estimator for Gerber-Shiu function and obtained its asymptotic property. Please refer to Equation (1.2) in [17] for the details of Gerber-Shiu function.

In our work, we suppose that a sample $\left\{X_{t_{1}^{n}}, X_{t_{2}^{n}}, X_{t_{3}^{n}}, \ldots, X_{t_{n}^{n}}\right\}$ can be observed, where $h_{n}=t_{i}^{n}-$ $t_{i-1}^{n}$ is the sampling interval. However, we cannot observe the exact time and size of claims. To estimate $\Phi(x)$, we have to estimate $F, \lambda, \lambda \mu_{\gamma}$ and $\sigma^{2}$. Given the discrete record of observations, we need to judge whether a claim occurs in the interval $\left(t_{i-1}^{n}, t_{i}^{n}\right]$. The threshold method from [19-22] is to determine that a single jump has occurred within $\left(t_{i-1}^{n}, t_{i}^{n}\right]$ if and only if the increment $\left|\Delta_{i} X\right|=\left|X_{t_{i}^{n}}-X_{t_{i-1}^{n}}\right|$ is larger than a suitable threshold function. By the threshold method and the work in [21,22], we can estimate $F, \lambda, \mu_{\gamma}$ and $\sigma^{2}$.

In $[14,17]$, the authors estimated the ruin probability and Gerber-Shiu function by a regularized Laplace inversion technique. Using the threshold method and the work in [23], it is easy to obtain an estimator for the Laplace transform of $\Phi(x)$. To estimate $\Phi(x)$, the regularized Laplace inversion technique is used. Finally, we also obtain a rate of convergence for the estimator of $\Phi(x)$ in a sense of the integrated squared error (ISE).

This paper is organized as follows. In Section 2, we give some estimators for $\sigma^{2}, \lambda \mu_{\gamma}, \lambda, F$ and its Laplace-Stieltjes transform. In Section 3, we study the asymptotic properties for the estimators. Finally, we give some conclusions in Section 5. All the technical proofs are presented in Appendix A.

\section{Estimation of Survival Probability}

To give the estimators for $\sigma^{2}, \lambda \mu_{\gamma}, \lambda, F$ and the Laplace transform of $F$, we introduce the following filter:

$$
\mathcal{C}_{i}^{n}\left(\vartheta\left(h_{n}\right)\right)=\left\{\omega \in \Omega ;\left|\Delta_{i} X(\omega)\right|>\vartheta\left(h_{n}\right)\right\},
$$

where $\vartheta\left(h_{n}\right)$ is a threshold function and $\mathcal{D}_{i}^{n}\left(\vartheta\left(h_{n}\right)\right)$ is a complement of $\mathcal{C}_{i}^{n}\left(\vartheta\left(h_{n}\right)\right)$. In [19,20], the threshold function $\vartheta\left(h_{n}\right)$ satisfies $\lim _{h_{n} \rightarrow 0} \vartheta\left(h_{n}\right)=0$ and $\lim _{h_{n} \rightarrow 0} \frac{\sqrt{h_{n} \log \left(\frac{1}{h_{n}}\right)}}{\vartheta\left(h_{n}\right)}=0$. In [21], the author gave an expression of threshold function $\vartheta\left(h_{n}\right)=L h_{n}^{b}$, where $L>0$ is a constant and $b \in\left(0, \frac{1}{2}\right)$. Obviously, the expression of $\vartheta\left(h_{n}\right)$ from [21] satisfies the two conditions. In our work, the expression of $\vartheta\left(h_{n}\right)$ is similar to that in [21].

We first estimate $F$. Using $\left\{\left|\Delta_{i} X\right| ; 0 \leq i \leq n, \mathbf{I}_{\mathcal{C}_{i}^{n}\left(\vartheta\left(h_{n}\right)\right)}=1\right\}$ and empirical distribution function, we can try to construct an estimator of $F$ as follows:

$$
\hat{F}_{n}(u)=\frac{1}{\sum_{i=1}^{n} \mathbf{I}_{i}^{n}\left(\vartheta\left(h_{n}\right)\right)} \sum_{i=1}^{n} \mathbf{I}_{\left\{\left|\Delta_{i} X\right| \leq u\right\}} \mathbf{I}_{\mathcal{C}_{i}^{n}\left(\vartheta\left(h_{n}\right)\right)}, \quad u \geq 0
$$

By Equations (3.4) and (3.6) in [21], the estimators of $\sigma^{2}$ and $\lambda$ are

$$
\widetilde{\sigma^{2}}{ }_{n}=\frac{\sum_{i=1}^{n}\left|\Delta_{i} X-c h_{n}\right|^{2} \mathbf{I}_{\mathcal{D}_{i}^{n}\left(\vartheta\left(h_{n}\right)\right)}}{T_{n}}, \quad \widetilde{\lambda}_{n}=\frac{\sum_{i=1}^{n} \mathbf{I}_{\mathcal{C}_{i}^{n}\left(\vartheta\left(h_{n}\right)\right)}}{T_{n}} .
$$


By Equation (3.10) in [21], an estimator of $\lambda \mu_{\gamma}$ is given by

$$
\widetilde{\lambda \mu_{\gamma_{n}}}=\frac{\sum_{i=1}^{n}\left|\Delta_{i} X\right| \mathbf{I}_{\mathcal{C}_{i}^{n}\left(\vartheta\left(h_{n}\right)\right)}}{T_{n}}
$$

Let $\rho=\frac{\lambda \mu_{\gamma}}{c}$. Obviously, the estimator of $\rho$ is given by

$$
\widetilde{\rho}_{n}=\frac{1}{c} \frac{\sum_{i=1}^{n}\left|\Delta_{i} X\right| \mathbf{I}_{\mathcal{C}_{i}^{n}\left(\vartheta\left(h_{n}\right)\right)}}{T_{n}} .
$$

The Laplace transform of $F$ is defined by $l_{F}=E\left[e^{-s \gamma_{1}}\right]=\int_{0}^{\infty} e^{-s u} F(d u)$. An estimator of $l_{F}$ is given by

$$
\widetilde{l}_{F n}(s)=\frac{\sum_{i=1}^{n} e^{-s\left|\Delta_{i} X\right|} \mathbf{I}_{\mathcal{C}_{i}^{n}\left(\vartheta\left(h_{n}\right)\right)},}{\sum_{i=1}^{n} \mathbf{I}_{\mathcal{C}_{i}^{n}\left(\vartheta\left(h_{n}\right)\right)}}
$$

where $s \in \mathbb{E}$ and $\mathbb{E}$ is a compact subset of $(0, \infty)$.

By the work in [23], the Laplace transform of $\Phi(x)$ can be obtained as follows:

$$
\begin{aligned}
L_{\Phi}(s) & =\int_{0}^{\infty} e^{-s x} \Phi(x) d x \\
& =\frac{1-\rho}{D(s)}, \quad s>0
\end{aligned}
$$

where $\rho=\frac{\lambda \mu}{c}$ and $D(s)=s+\frac{\sigma^{2}}{2 c} s^{2}-\frac{\lambda}{c}\left(1-l_{F}(s)\right)$.

Let us define an estimator of $L_{\Phi}(s)$ as follows:

$$
\widetilde{L_{\Phi}}(s)=\frac{1-\widetilde{\rho}_{n}}{\widetilde{D}(s)}, \quad \widetilde{D}(s)=s+\frac{\widetilde{\sigma}^{2} n}{2 c} s^{2}-\frac{\widetilde{\lambda}_{n}}{c}\left(1-{\widetilde{l_{F}}}_{n}(s)\right), \quad s>0 .
$$

To estimate $\Phi(x)$, we use the $L^{2}$-inversion method proposed from [24]. Now, we give the $L^{2}$-inversion method by Definition 1 . We say that $f \in L^{2}(0, \infty)$ if $\left(\int_{0}^{\infty}|f(t)|^{2} d t\right)^{\frac{1}{2}}<\infty$.

Definition 1. Let $m>0$ be a constant. The regularized Laplace inversion $L_{m}^{-1}: L^{2}(0, \infty) \rightarrow L^{2}(0, \infty)$ is given by

$$
L_{m}^{-1} g(t)=\frac{1}{\pi^{2}} \int_{0}^{\infty} \int_{0}^{\infty} \Psi_{m}(y) y^{-\frac{1}{2}} e^{-t v y} g(v) d v d y
$$

for a function $g \in L^{2}(0, \infty)$ and $t \in(0, \infty)$, where

$$
\Psi_{m}(y)=\int_{0}^{a_{m}} \cosh (\pi x) \cos (x \log y) d x
$$

and $a_{m}=\pi^{-1} \cosh ^{-1}(\pi m)>0$.

For further information, and details of $L_{m}^{-1}$, please refer to [24].

To use Definition 1 , it requires to verify $\widetilde{L_{\Phi}}(s) \in L^{2}(0, \infty)$. As $n$ is sufficiently large, for P-almost all $\omega \in \Omega$ and $s>0$, we have

$$
\mathbf{P}\left(\left\{\omega \in \Omega ;\left(1-\widetilde{\rho}_{n}\right) s \leq \widetilde{D}(s) \leq s+\frac{\widetilde{\sigma^{2}} n}{2 c} s^{2}\right\}\right)=1 .
$$

From Equations (6) and (8), it is obvious that $\widetilde{L_{\Phi}}(s) \notin L^{2}(0, \infty)$. The $L^{2}$-inversion method in Definition 1 cannot be applied at once. 
Therefore, to use Definition 1 , we have to amend $\widetilde{L_{\Phi}}(s)$.

Let

$$
\Phi_{\theta}(x)=e^{-\theta x} \Phi(x), \quad x>0
$$

for arbitrary fixed $\theta>0$. It is obvious that

$$
L_{\Phi_{\theta}}(s)=L_{\Phi}(s+\theta), \quad s>0 .
$$

An estimator of $L_{\Phi_{\theta}}$ is given by

$$
\widetilde{L_{\Phi_{\theta}}}(s)=\widetilde{L_{\Phi}}(s+\theta), \quad s>0 .
$$

Obviously, $\widetilde{L_{\Phi_{\theta}}} \in L^{2}(0, \infty)$.

Finally, an estimator of $\Phi(x)$ is given by

$$
\widetilde{\Phi}_{m(n)}(x)=e^{\theta x} \widetilde{\Phi_{\theta, m(n)}}(x), \quad x>0,
$$

where $\widetilde{\Phi_{\theta, m(n)}}(x)=L_{m(n)}^{-1} \widetilde{L_{\Phi_{\theta}}}(s)$ and $m(n)>0$.

\section{Asymptotic Properties}

According to Theorem 3.1 in [19], the author assumed that $\sigma<Q$ and $\gamma_{i} \geq \Gamma$ with $Q>0, \Gamma>0$. In our work, Assumption 1 is used to prove the asymptotic properties of estimators.

Assumption 1. There exist two positive constants $Q$ and $\Gamma$ such that $\sigma<Q$ and $\mathbf{P}\left(\left\{\omega \in \Omega ; \gamma_{i} \geq \Gamma\right\}\right)=1$ for $i=1,2, \ldots$.

Let $\bar{F}=1-F$. With Equation (4), an estimator of $\bar{F}$ is given by

$$
\overline{\hat{F}}_{n}(u)=\frac{1}{\sum_{i=1}^{n} \mathbf{I}_{\mathcal{C}_{i}^{n}\left(\vartheta\left(h_{n}\right)\right)}} \sum_{i=1}^{n} \mathbf{I}_{\left\{\left|\Delta_{i} X\right|>u\right\}} \mathbf{I}_{\mathcal{C}_{i}^{n}\left(\vartheta\left(h_{n}\right)\right)}
$$

Let $\mathcal{N}(m, n)$ be a normal distribution with expectation $m$ and variance $n$. Theorem 1 gives the asymptotic properties of $\hat{F}_{n}(u)$.

Theorem 1. Suppose that $T_{n}=n h_{n} \rightarrow \infty, n h_{n}^{2} \rightarrow 0, h_{n} \rightarrow 0$ as $n \rightarrow \infty$ and Assumption 1 is satisfied, then

$$
\sqrt{T_{n}}\left(\overline{\hat{F}}_{n}(u)-\bar{F}(u)\right) \stackrel{\mathbf{D}}{\rightarrow} \mathcal{N}\left(0, \frac{\bar{F}(u)(1-\bar{F}(u))}{\lambda}\right) .
$$

Obviously,

$$
\sqrt{T_{n}}\left(\hat{F}_{n}(u)-F(u)\right) \stackrel{\mathbf{D}}{\rightarrow} \mathcal{N}\left(0, \frac{F(u)(1-F(u))}{\lambda}\right) .
$$

Remark 1. By Dvoretzky-Kiefer-Wolfowitz inequality, we have

$$
\mathbf{P}\left(\sup _{u \in[0, \infty)}\left|\hat{F}_{n}(u)-F(u)\right|>x\right) \leq C e^{-2 \lambda T_{n} x^{2}}, \quad x>0,
$$

where $C$ is a positive constant, not depending on $F$. Note that this inequality may be expression in the form:

$$
\mathbf{P}\left(\sqrt{T_{n}} \sup _{u \in[0, \infty)}\left|\hat{F}_{n}(u)-F(u)\right|>x\right) \leq C e^{-2 \lambda x^{2}}, \quad x>0,
$$


which clearly demonstrate that

$$
\sqrt{T_{n}} \sup _{u \in[0, \infty)}\left|\hat{F}_{n}(u)-F(u)\right|=O_{\mathbf{P}}(1) .
$$

The asymptotic properties of $\widetilde{\sigma^{2}}{ }_{n}$ are given by the following Lemma 1.

Lemma 1. Suppose that $T_{n}=n h_{n} \rightarrow \infty, n h_{n}^{2} \rightarrow 0$ and $h_{n} \rightarrow 0$ as $n \rightarrow \infty$, then

$$
\begin{gathered}
{\widetilde{\sigma^{2}}}_{n} \stackrel{\mathbf{P}}{\rightarrow} \sigma^{2}, \quad n \rightarrow \infty . \\
\sqrt{n}\left({\widetilde{\sigma^{2}}}_{n}-\sigma^{2}\right) \stackrel{\mathbf{D}}{\rightarrow} \mathcal{N}\left(0,2 \sigma^{4}\right), \quad n \rightarrow \infty .
\end{gathered}
$$

Lemma 2. Suppose that $T_{n}=n h_{n} \rightarrow \infty, n h_{n}^{\beta} \rightarrow 0$ for some $\beta \in(1,2], h_{n} \rightarrow 0$ as $n \rightarrow \infty$ and Assumption 1 is satisfied. Then,

$$
\begin{gathered}
\widetilde{\lambda}_{n} \stackrel{\mathbf{P}}{\rightarrow} \lambda, \sup _{\{s \mid s \in \mathbb{E}\}}\left|\widetilde{\lambda}_{n} \widetilde{l}_{F n}(s)-\lambda l_{F}(s)\right| \stackrel{\mathbf{P}}{\rightarrow} 0, \\
\sqrt{T_{n}}\left(\widetilde{\lambda}_{n}-\lambda\right) \stackrel{\mathbf{D}}{\rightarrow} \mathcal{N}(0, \lambda), \\
\widetilde{\rho}_{n} \stackrel{\mathbf{P}}{\rightarrow} \rho
\end{gathered}
$$

and

$$
\sqrt{n}\left(\widetilde{\rho}_{n}-\rho\right) \stackrel{\mathbf{D}}{\rightarrow} \mathcal{N}\left(0, \frac{\lambda \sigma^{2}}{c^{2}}\right)
$$

as $n \rightarrow \infty$.

Let $\|f\|_{B}^{2}=\int_{0}^{B}|f(t)|^{2} d t$ for any function $f$ and $B>0$. Theorem 2 gives a rate of convergence for $\widetilde{\Phi}_{m(n)}(x)$ in a sense of ISE.

Theorem 2. Suppose that there exists a constant $K>0$ such that $0 \leq \Phi^{\prime}(x)=g(x) \leq K<\infty$ and the conditions in Lemma 2 are satisfied. Then, for $m(n)=\sqrt{\frac{T_{n}}{\log T_{n}}}$ and for any constant $B>0$, we have

$$
\left\|\widetilde{\Phi}_{m(n)}-\Phi\right\|_{B}^{2}=O_{P}\left(\left(\log T_{n}\right)^{-1}\right), \quad n \rightarrow \infty .
$$

Remark 2. The explicit expression for $\widetilde{\Phi}_{m(n)}(x)$ is

$$
\widetilde{\Phi}_{m(n)}(x)=\frac{e^{x \theta}}{\pi^{2}} \int_{0}^{\infty} \int_{0}^{\infty} e^{-x s y} \widetilde{L_{\Phi_{\theta}}}(s) \Psi_{m(n)}(y) y^{-\frac{1}{2}} d s d y
$$

where $\Psi_{m(n)}(y)=\int_{0}^{a_{m(n)}} \cosh (\pi x) \cos (x \log (y)) d x$ and $a_{m(n)}=\pi^{-1} \cosh ^{-1}(\pi m(n))>0$ and $m(n)=\sqrt{\frac{T_{n}}{\log T_{n}}}$.

When $c, \lambda, \sigma, F, \theta, \vartheta\left(h_{n}\right)$ and sample size $n$ are known, $\widetilde{\Phi}_{m(n)}(x)$ can be evaluated with the command integral $2(f ; 0 ; \infty ; 0 ; \infty)$ of Matlab.

\section{Simulation}

If $F(x)=1-e^{-\frac{1}{\mu_{\gamma}} x}$, the survival probability is given by

$$
\Phi(x)=1-\frac{r_{1}+\frac{1}{\mu_{\gamma}}+\frac{2 \lambda \mu_{\gamma}}{\sigma^{2}}}{r_{1}-r_{2}} e^{r_{1} x}-\frac{r_{2}+\frac{1}{\mu_{\gamma}}+\frac{2 \lambda \mu_{\gamma}}{\sigma^{2}}}{r_{2}-r_{1}} e^{r_{2} x}, \quad x \geq 0,
$$


where $r_{2}<r_{1}<0$ are negative roots of the following equation

$$
\frac{1}{2} \sigma^{2} s+c-\frac{\lambda}{s+\frac{1}{\mu_{\gamma}}}=0
$$

By the work in [25], Equation (18) is obtained easily.

Let $c=\lambda=10, \mu_{\gamma}=\frac{1}{2}, \sigma=5, \theta=0.075, \vartheta\left(h_{n}\right)=h_{n}^{b}, b=\frac{1}{4}$ and $h_{n}=n^{-\frac{4}{5}}$.

Firstly, we computed $\widetilde{L_{\Phi_{\theta}}}(s)$. In Figure 1, we plot the mean points with sample sizes $n=5000$, $10,000,30,000,50,000,80,000$, which were computed based on 5000 simulation experiments.

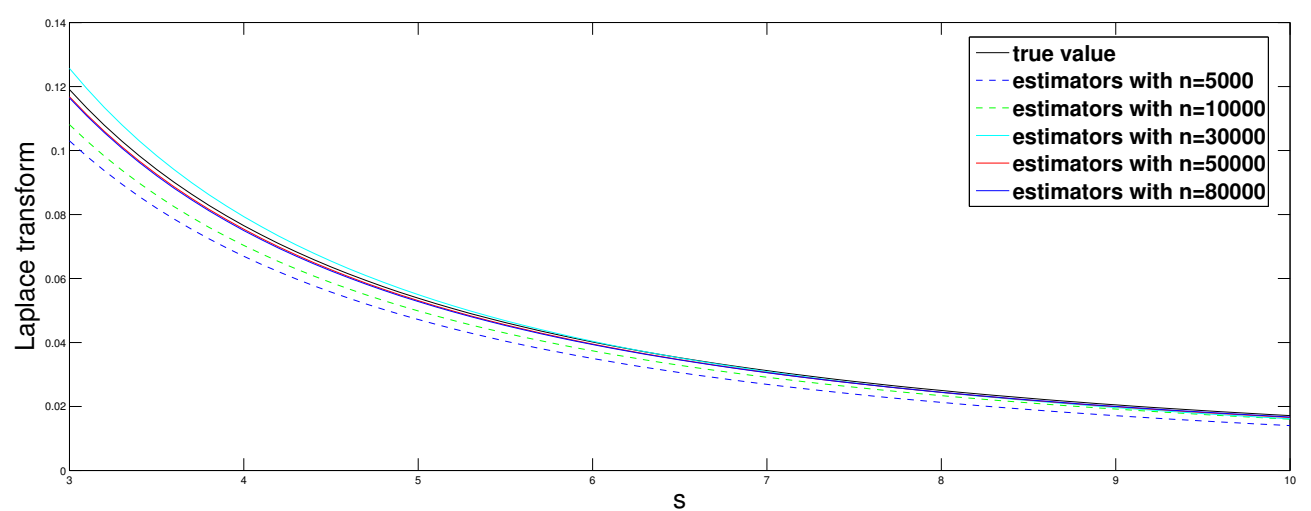

Figure 1. The estimator of $\mathcal{L}_{\Phi_{\theta}}$ with sample sizes $n=5000,10,000,30,000,50,000,80,000$.

In Remark 2, $\widetilde{\Phi}_{m(n)}(u)$ is a double complex integrals. Using Matlab to compute $\widetilde{\Phi}_{m(n)}(u)$ would take a long time. As shown in Figure $1, \hat{\mathcal{L}}_{\Phi_{\theta}}$ is very close to $\mathcal{L}_{\Phi_{\theta}}$ as $n \geq 30,000$. To improve computational efficiency, let

$$
\Phi_{p}(x)=\frac{e^{x \theta}}{\pi^{2}} \int_{0}^{\infty} \int_{0}^{\infty} e^{-x s y}\left[\hat{\mathcal{L}}_{\Phi_{\theta}}(s)\right]_{n=30000} \Psi_{p}(y) y^{-\frac{1}{2}} d s d y,
$$

where $\left[\hat{\mathcal{L}}_{\Phi_{\theta}}(s)\right]_{n=30000}=\frac{1-\frac{1}{c 30000(30000)^{-\frac{4}{5}}} \sum_{k=1}^{30000}\left(c(30000)^{-\frac{4}{5}}-Z_{k}\right) \mathbf{I}_{\mathcal{D}_{k}^{30000}}}{\frac{1}{c(30000)^{-\frac{4}{5}}}\left(\frac{1}{30000} \sum_{k=1}^{30000} e^{\left.s Z_{k}-1\right)}\right.}, \Psi_{p}(y)=\int_{0}^{a_{p}} \cosh (\pi x) \cos (x \log (y)) d x$ and $a_{p}=\pi^{-1} \cosh ^{-1}(\pi p)>0$.

In Figure 2, we plot the mean points with sample sizes $n=30,000$ and $p=100,500,800,1000,3000$, which were computed based on 5000 simulation experiments.

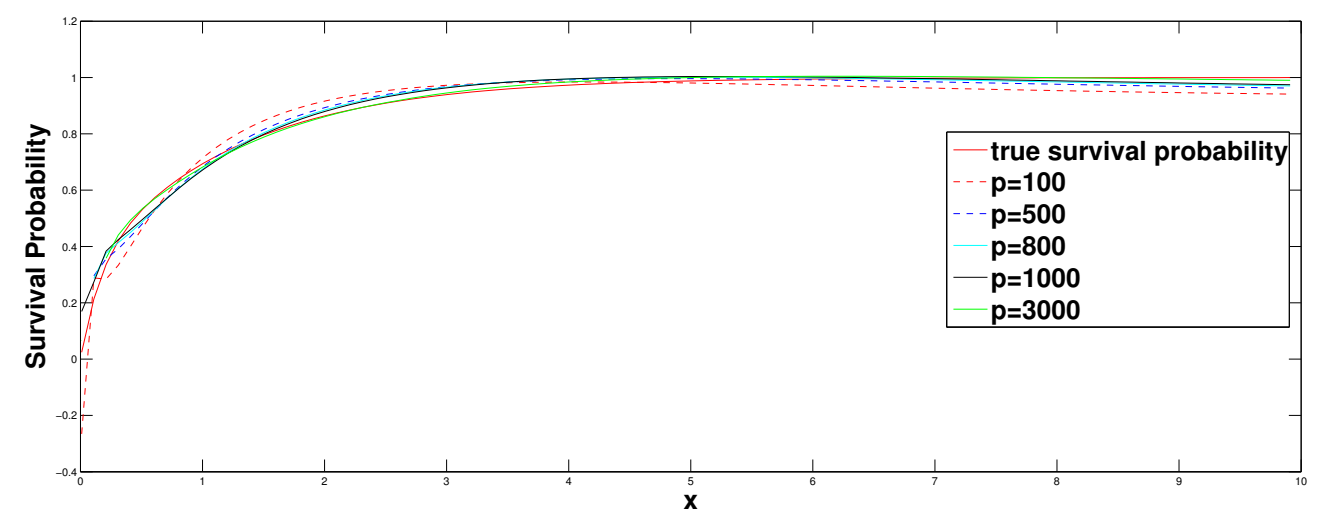

Figure 2. $\Phi_{p}(x)$ with sample size $n=30,000$ and $p=100,500,800,1000,3000$ 


\section{Conclusions}

In this paper, we use the threshold estimation technique and regularized Laplace inversion technique to constructed an estimator of survival probability for the Wiener-Poisson risk model. The rate of convergence for the estimator is a logarithmic rate. We adopt a method proposed by Cai et al. [26] to improve the speed in simulated calculation. The further work is to improve the speed of convergence for the estimator. We will combine the threshold estimation technique with Fourier transform (inversion) technique to construct an estimator of survival probability. We hope some further studies will be done when the risk model is the compound Poisson model with the barrier dividend strategy and investment. The Gerber-Shiu function and dividend function will be estimated by some statistical methods.

Author Contributions: Methodology, H.Y.; Formal analysis, H.Y. and Y.G.; Simulation, H.Y.; Writing-original draft, H.Y.

Funding: This research was partially supported by the National Natural Science Foundation of China (Grant Nos. 11571189, 11571198, 11501319 and 11701319), the Postdoctoral Science Foundation of China (Grant No. 2018M642634) and the Higher Educational Science and Technology Program of Shandong Province of China (Grant No. J15LI05).

Acknowledgments: The authors would like to express their thanks to three anonymous referees for their helpful comments and suggestions, which improved an earlier version of the paper.

Conflicts of Interest: The authors declare no conflicts of interest.

\section{Appendix A. Proofs of Theorems}

Proof of Lemma 1. The proof of Lemma 1 is easily obtained by Theorem 3.1 in [21].

Proof of Lemma 2. The proof of Equation (14) is given as Theorem 3.2 in [21]. By Theorem 3.1 in [19], we can get Equation (15). It is easy to get Equations (16) and (17) by Proposition 3.4 in [19] and Theorem 3 in [20].

To prove Theorem 1, we need the following Proposition, which can be easily obtained in Section 3.2 of [19].

Proposition A1. Following from the condition of Theorem 1, for any $\epsilon>0$,

$$
\lim _{n \rightarrow \infty} P\left(\left|\Delta_{i} X\right| \mathbf{I}_{\mathcal{C}_{i}^{n}\left(\vartheta\left(h_{n}\right)\right)}-\gamma_{\tau^{(i)}} \mathbf{I}_{\left\{\Delta_{i} N \geq 1\right\}} \mid>\epsilon\right)=0,
$$

where $\gamma_{\tau^{(i)}}$ is the size of the eventual jump in time interval $\left(t_{i-1}^{n}, t_{i}^{n}\right]$.

Proof of Theorem 1. By Equation (10),

$$
\sqrt{T_{n}}\left(\overline{\hat{F}}_{n}(u)-\bar{F}(u)\right)=\frac{J}{\widetilde{\lambda}_{n}},
$$

where

$$
J=\frac{\sum_{i=1}^{n}\left(\mathbf{I}_{\left\{\left|\Delta_{i} X\right|>u\right\}}-\bar{F}(u)\right) \mathbf{I}_{\mathcal{C}_{i}^{n}\left(\vartheta\left(h_{n}\right)\right)}}{\sqrt{T_{n}}} .
$$

As $n \rightarrow \infty$, the expectation of $J$ is

$$
\begin{aligned}
\lim _{n \rightarrow \infty} \mathbf{E}[J] & =\lim _{n \rightarrow \infty} \frac{n}{\sqrt{T_{n}}} \mathbf{E}\left[\left(\mathbf{I}_{\left\{\left|\Delta_{i} X\right|>u\right\}}-\bar{F}(u)\right) \mathbf{I}_{\mathcal{C}_{i}^{n}\left(\vartheta\left(h_{n}\right)\right)}\right] \\
& =\lim _{n \rightarrow \infty} \frac{n}{\sqrt{T_{n}}}\left[\mathbf{P}\left(\left|\Delta_{i} X\right| \mathbf{I}_{\mathcal{C}_{i}^{n}\left(\vartheta\left(h_{n}\right)\right)}>u\right)-\bar{F}(u) \mathbf{P}\left(\left|\Delta_{i} X\right|>\vartheta\left(h_{n}\right)\right)\right] .
\end{aligned}
$$


By Proposition A1, we have

$$
\lim _{n \rightarrow \infty} \mathbf{P}\left(\left|\Delta_{i} X\right| \mathbf{I}_{\mathcal{C}_{i}^{n}\left(\vartheta\left(h_{n}\right)\right)}>u\right)=\lim _{n \rightarrow \infty} \mathbf{P}\left(\gamma_{\tau^{(i)}} \mathbf{I}_{\left\{\Delta_{i} N \geq 1\right\}}>u\right)=\bar{F}(u)\left(\lambda h_{n}+o\left(h_{n}\right)\right) .
$$

By the work in [19], it is obvious that

$$
\lim _{n \rightarrow \infty} \mathbf{P}\left(\left|\Delta_{i} X\right|>\vartheta\left(h_{n}\right)\right)=\lambda h_{n}+o\left(h_{n}\right) .
$$

Therefore,

$$
\lim _{n \rightarrow \infty} \mathbf{E}[J]=0
$$

As $n \rightarrow \infty$, the variance of $J$ is

$$
\begin{aligned}
\lim _{n \rightarrow \infty} \operatorname{Var}[J]= & \lim _{n \rightarrow \infty} \frac{n}{T_{n}} \operatorname{Var}\left[\left(\mathbf{I}_{\left\{\left|\Delta_{i} X\right|>u\right\}}-\bar{F}(u)\right) \mathbf{I}_{\mathcal{C}_{i}^{n}\left(\vartheta\left(h_{n}\right)\right)}\right] \\
= & \lim _{n \rightarrow \infty} \frac{n}{T_{n}} \mathbf{E}\left[\mathbf{I}_{\left\{\left|\Delta_{i} X\right| \mathbf{I}_{\mathcal{C}_{i}^{n}\left(\vartheta\left(h_{n}\right)\right)}>u\right\}}\right]+\lim _{n \rightarrow \infty} \frac{n}{T_{n}} \mathbf{E}\left[(\bar{F}(u))^{2} \mathbf{I}_{\mathcal{C}_{i}^{n}\left(\vartheta\left(h_{n}\right)\right)}\right] . \\
& -\lim _{n \rightarrow \infty} \frac{n}{T_{n}} \mathbf{E}\left[2 \bar{F}(u) \mathbf{I}_{\left\{\left|\Delta_{i} X\right| \mathbf{I}_{\mathcal{C}_{i}^{n}\left(\vartheta\left(h_{n}\right)\right)}>u\right\}}\right] .
\end{aligned}
$$

By Equations (A3) and (A4),

$$
\lim _{n \rightarrow \infty} \operatorname{Var}[J]=\lambda \bar{F}(u)(1-\bar{F}(u)) .
$$

With the central limit theorem, Slutsky's theorem and Lemma 2, we have

$$
\sqrt{T_{n}}\left(\overline{\hat{F}}_{n}(u)-\bar{F}(u)\right) \stackrel{\mathbf{D}}{\rightarrow} \mathcal{N}\left(0, \frac{\bar{F}(u)(1-\bar{F}(u))}{\lambda}\right), \quad n \rightarrow \infty .
$$

To prove Theorem 2, we need the following Lemma A1.

Lemma A1. Suppose that $\int_{0}^{\infty}\left[t\left(t^{\frac{1}{2}} f(t)\right)^{\prime}\right]^{2} t^{-1} d t<\infty$ for a function $f \in L^{2}(0, \infty)$ with the derivative $f^{\prime}$. Then,

$$
\left\|L_{n}^{-1} L_{f}-f\right\|=O\left((\log n)^{-\frac{1}{2}}\right), \quad n \rightarrow \infty .
$$

By Theorem 3.2 in [24], the proof of Lemma A1 can be found.

Proof of Theorem 2. By Equation (9),

$$
\begin{aligned}
\left\|\widetilde{\Phi}_{m(n)}-\Phi\right\|_{B}^{2} & \leq e^{2 \theta B}\left\|\widetilde{\Phi}_{\theta, m(n)}-\Phi_{\theta}\right\|_{B}^{2} \\
& \leq 2 e^{2 \theta B}\left\{\left\|L_{m(n)}^{-1} \widetilde{L_{\Phi_{\theta}}}-L_{m(n)}^{-1} L_{\Phi_{\theta}}\right\|^{2}+\left\|\Phi_{\theta, m(n)}-\Phi_{\theta}\right\|^{2}\right\} .
\end{aligned}
$$


Let $\Phi_{\theta}^{\prime}=g_{\theta}$. Now, we show that $\Phi_{\theta, m(n)}$ satisfies the condition of Lemma A1.

$$
\begin{aligned}
\int_{0}^{\infty}\left[x\left(\sqrt{x} \Phi_{\theta}(x)\right)^{\prime}\right]^{2} \frac{1}{x} d x & =\int_{0}^{\infty}\left[x\left(\frac{1}{2 \sqrt{x}} \Phi_{\theta}(x)+x \sqrt{x} g_{\theta}(x)\right)\right]^{2} \frac{1}{x} d x \\
& \leq \int_{0}^{\infty} 2 \frac{1}{x}\left[x \frac{1}{2 \sqrt{x}} \Phi_{\theta}(x)\right]^{2}+\int_{0}^{\infty} 2 \frac{1}{x}\left[x \sqrt{x} g_{\theta}(x)\right]^{2} d x \\
& =\int_{0}^{\infty} \frac{1}{2} \Phi_{\theta}^{2}(x) d x+2 \int_{0}^{\infty} x^{2} g_{\theta}^{2}(x) d x \\
& \leq \int_{0}^{\infty} \frac{1}{2} e^{-2 \theta x} d x+2 \int_{0}^{\infty} x^{2}\left[g(x) e^{-\theta x}-\theta \Phi(x) e^{-\theta x}\right]^{2} d x \\
& \leq \frac{1}{4 \theta}+4 \int_{0}^{\infty} x^{2} g^{2}(x) e^{-2 \theta x} d x+4 \theta^{2} \int_{0}^{\infty} \Phi^{2}(x) x^{2} e^{-2 \theta x} d x \\
& \leq \frac{1}{4 \theta}+4\left(K^{2}+\theta^{2}\right) \int_{0}^{\infty} x^{2} e^{-2 \theta x} d x<\infty .
\end{aligned}
$$

Therefore, by Lemma A1, we have

$$
\left\|\Phi_{\theta, m(n)}-\Phi_{\theta}\right\|^{2}=O\left(\frac{1}{\log m(n)}\right), \quad n \rightarrow \infty .
$$

By Equations (5) and (6),

$$
\left\|\widetilde{L_{\Phi_{\theta}}}-L_{\theta}\right\|^{2}=\int_{0}^{\infty}\left(\frac{(1-\rho)(\widetilde{D}(s+\theta)-D(s+\theta))}{\widetilde{D}(s+\theta) D(s+\theta)}+\frac{\left(\widetilde{\rho}_{n}-\rho\right)}{\widetilde{D}(s+\theta)}\right)^{2} d s .
$$

Exploiting Equation (8) and $\mathbf{P}\left(\left\{\omega \in \Omega ; \widetilde{\rho}_{n}=1\right\}\right)=0$, the right-hand side of Equation (A7) is bounded by

$$
2 \int_{0}^{\infty} \frac{(\widetilde{D}(s+\theta)-D(s+\theta))^{2}}{(s+\theta)^{4}\left(1-\widetilde{\rho}_{n}\right)^{2}} d s+2 \int_{0}^{\infty}\left(\frac{\widetilde{\rho}_{n}-\rho}{1-\widetilde{\rho}_{n}}\right)^{2} \frac{1}{(s+\theta)^{2}} d s
$$

By Lemmas 1 and 2, the term

$$
\begin{aligned}
\widetilde{D_{n}}(s+\theta)-D(s+\theta) & =\frac{(s+\theta)^{2}}{2 c}\left(\widetilde{\sigma}^{2}{ }_{n}-\sigma^{2}\right)+\frac{1}{c}\left(\left(\lambda-\widetilde{\lambda}_{n}\right)+\left(\widetilde{\lambda}_{n}{\widetilde{l_{F}}}_{n}(s+\theta)-\lambda l_{F}(s+\theta)\right)\right) \\
& =O_{\mathbf{P}}\left(T_{n}^{-\frac{1}{2}}\right)+\frac{1}{c} \frac{N_{T_{n}}}{T_{n}}\left(\int_{0}^{\infty} e^{-(s+\theta) x}\left(\hat{F}_{n}(d x)-F(d x)\right)\right) \\
& =O_{\mathbf{P}}\left(T_{n}^{-\frac{1}{2}}\right)+\frac{1}{c} \frac{N_{T_{n}}}{T_{n}}\left(\int_{0}^{\infty}(s+\theta) e^{-(s+\theta) x}\left(\hat{F}_{n}(x)-F(x)\right) d x\right) \\
& \leq O_{\mathbf{P}}\left(T_{n}^{-\frac{1}{2}}\right)+\frac{1}{c} \frac{N_{T_{n}}}{T_{n}} \sup _{x \in[0, \infty)}\left|\hat{F}_{n}(x)-F(x)\right| \\
& =O_{\mathbf{P}}\left(T_{n}^{-\frac{1}{2}}\right)
\end{aligned}
$$

The last equality is obtained from Remark 1.

By Equation (A9) and Lemma 2, we have

$$
2 \int_{0}^{\infty} \frac{(\widetilde{D}(s+\theta)-D(s+\theta))^{2}}{(s+\theta)^{4}\left(1-\widetilde{\rho}_{n}\right)^{2}} d s=O_{\mathbf{P}}\left(T_{n}^{-1}\right)
$$

and

$$
2 \int_{0}^{\infty}\left(\frac{\widetilde{\rho}_{n}-\rho}{1-\widetilde{\rho}_{n}}\right)^{2} \frac{1}{(s+\theta)^{2}} d s=o_{\mathbf{P}}\left(T_{n}^{-1}\right)
$$


Recall that $\left\|L_{m(n)}^{-1}\right\|^{2} \leq \pi m^{2}(n)$ (see [24]), so

$$
\left\|L_{m(n)}^{-1}\right\|^{2}\left\|\widetilde{L_{\Phi_{\theta}}}-L_{\Phi_{\theta}}\right\|^{2}=O_{\mathbf{P}}\left(\frac{m^{2}(n)}{T_{n}}\right) .
$$

Combining Equations (A6) and (A10), we have

$$
\left\|\widetilde{\Phi}_{m(n)}-\Phi\right\|_{B}^{2}=O_{\mathbf{P}}\left(\frac{m^{2}(n)}{T_{n}}\right)+O_{\mathbf{P}}\left(\frac{1}{\log m(n)}\right) .
$$

with an optimal $m(n)=\sqrt{\frac{T_{n}}{\log T_{n}}}$ balancing the the two right-hand terms in Equation (A11), the order becomes $O_{\mathbf{P}}\left(\left(\log T_{n}\right)^{-1}\right)$.

\section{References}

1. Gerber, H.U. An extension of the renewal equation and its application in the collective theory of risk. Scand. Actuar. J. 1970, 1970, 205-210. [CrossRef]

2. Dufresne, F.; Gerber, H.U. Risk theory for the compound Poisson process that is perturbed by diffusion. Insur. Math. Econ. 1991, 10, 51-59. [CrossRef]

3. Furrer, H.J.; Schimidli, H. Exponential inequalities for ruin probabilities of risk process perturbed by diffusion. Insur. Math. Econ. 1994, 15, 23-36. [CrossRef]

4. Gatto, R.; Mosimann, M. Four approaches to compute the probability of ruin in the compound Poisson risk process with diffusion. Math. Comput. Modell. 2012, 55, 1169-1185. [CrossRef]

5. Gatto, R.; Baumgartner, B. Saddlepoint Approximations to the Probability of Ruin in Finite Time for the Compound Poisson Risk Process Perturbed by Diffusion. Methodol. Comput. Appl. Probab. 2014, 18, 1-19. [CrossRef]

6. Gatto, R. Importance sampling approximations to various probabilities of ruin of spectrally negative Lévy risk processes. Appl. Math. Comput. 2014, 243, 91-104. [CrossRef]

7. Schimidli, H. Cramer-Lundberg approximations for ruin probabilites of risk processes perturbed by diffusion. Insur. Math. Econ. 1995, 16, 135-149. [CrossRef]

8. Veraverbeke, N. Asymptotic estimates for the probability of ruin in a Poisson model with diffusion. Insur. Math. Econ. 1993, 13, 57-62. [CrossRef]

9. Wang, Y.; Yin, C. Approximation for the ruin probabilities in a discrete time risk model with dependent risks. Stat. Probab. Lett. 2010, 80, 1335-1342. [CrossRef]

10. Bening, V.E.; Korolev, V.Y. Nonparametric estimation of the ruin probability for generalized risk processes. Theory Probab. Its Appl. 2002, 47, 1-16. [CrossRef]

11. Croux, K.; Veraverbeke, N. Non-parametric estimators for the probability of ruin. Insur. Math. Econ. 1990, 9, 127-130. [CrossRef]

12. Frees, EW. Nonparametric estimation of the probability of ruin. Astin Bull. 1986, 16, 81-90. [CrossRef]

13. Hipp, C. Estimators and bootstrap confidence intervals for ruin probabilities. Astin Bull. 1989, 19, 57-70. [CrossRef]

14. Mnatsakanov, R.; Ruymgaart, L.L.; Ruymgaart, F.H. Nonparametric estimation of ruin probabilities given a random sample of claims. Math. Methods Stat. 2008, 17, 35-43. [CrossRef]

15. Pitts, S.M. Nonparametric estimation of compound distributions with applications in insurance. Ann. Inst. Stat. Math. 1994, 46, 537-555.

16. Politis, K. Semiparametric estimation for non-ruin probabilities. Scand. Actuar. J. 2003, 2003, 75-96. [CrossRef]

17. Shimizu, Y. Non-parametric estimation of the Gerber-Shiu function for the Wiener-Poisson risk model. Scand. Actuar. J. 2012, 2012, 56-69. [CrossRef]

18. Zhang, Z.; Yang, H. Nonparametric estimate of the ruin probability in a pure-jump lévy risk model. Insur. Math. Econ. 2013, 53, 24-35. [CrossRef]

19. Mancini, C. Estimation of the characteristics of the jump of a general Poisson-diffusion model. Scand. Actuar. J. 2004, 1, 42-52. [CrossRef] 
20. Mancini, C. Non-parametric threshold estimation for models with stochastic diffusion coefficient and jumps. Scand. J. Stat. 2009, 36, 270-296. [CrossRef]

21. Shimizu ,Y. A new aspect of a risk process and its statistical inference. Insur. Math. Econ. 2009, 44, 70-77. [CrossRef]

22. Shimizu, Y. Functional estimation for lévy measures of semimartingales with Poissonian jumps. J. Multivar. Anal. 2009, 100, 1073-1092. [CrossRef]

23. Morales, M. On the expected discounted penalty function for a perturbed risk process driven by a subordinator. Insur. Math. Econ. 2007, 40, 293-301. [CrossRef]

24. Chauveau, D.E.; Vanrooij, A.C.M.; Ruymgaart, F.H. Regularized inversion of noisy Laplace transforms. Adv. Appl. Math. 1994, 15, 186-201. [CrossRef]

25. Asmussen, S.; Albrecher, H. Ruin Probabilities, 2nd ed.; World Scientific: Singapore, 2010.

26. Cai, C.; Chen, N.; You, H. Nonparametric estimation for a spectrally negative Lévy process based on low-frequency observation. J. Comput. Appl. Math. 2018, 328, 432-442. [CrossRef]

(C) 2019 by the authors. Licensee MDPI, Basel, Switzerland. This article is an open access article distributed under the terms and conditions of the Creative Commons Attribution (CC BY) license (http:// creativecommons.org/licenses/by/4.0/). 